\title{
Preoperative Extrapontine Myelinolysis with Good Outcome in a Patient with Pituitary Adenoma
}

\author{
Ying Zhou, M.D., ${ }^{1}$ Yicheng Zhu, M.D., ${ }^{2}$ Wenze Wang, M.D., ${ }^{3}$ Bing Xing, M.D. ${ }^{1}$ \\ Departments of Neurosurgery, ${ }^{1}$ Neurology, ${ }^{2}$ Pathology, ${ }^{3}$ Peking Union Medical College Hospital, Chinese Academy of Medical Sciences, \\ Beijing, P.R. China
}

\begin{abstract}
Few preoperative extrapontine myelinolysis (EPM) cases with pituitary adenoma have been reported. No such case had long follow-up to see the outcome of EPM. We reported a 38-year-old man complaining of nausea, malaise and transient loss of consciousness who was found to have severe hyponatremia. Neurologic deficits including altered mental status, behavioral disturbances, dysarthria and dysphagia developed despite slow correction of hyponatremia. Endocrine and imaging studies revealed hypopituitarism, nonfunctional pituitary macroadenoma and extrapontine myelinolysis. Transsphenoidal surgery was performed after three weeks of supportive therapy, when neurological symptoms improved significantly. The patient recovered function completely 3 months after surgery. Our case indicates that outcome of EPM can be good even with prolonged periods of severe neurologic impairment.
\end{abstract}

Key Words : Extrapontine myelinolysis · Hyponatremia · Outcome · Pituitary adenoma · Preoperative.

\section{INTRODUCTION}

Osmotic demyelination syndrome (ODS) including central pontine myelinolysis (CPM) and extrapontine myelinolysis (EPM) following overly rapid correction of hyponatremia has been well recognized ${ }^{5,15)}$. Although ODS occurring after transsphenoidal resection of pituitary adenoma has been reported occasionally $^{11,16,18)}$, to our knowledge, few preoperative EPM cases with pituitary adenoma have been reported ${ }^{14)}$. Unfortunately, the outcomes of patients with ODS are often disappointing with high rate of mortality and irreversible neurological deficits $^{5}$. Here, we report a case of preoperative EPM with nonfunctional pituitary macroadenoma who made a great clinical recovery after supportive therapy and transsphenoidal adenoma resection.

\section{CASE REPORT}

A 38-year-old man was admitted to our hospital in December 2012 because of nausea, malaise, altered mental status, behavioral disturbances, dysarthria and dysphagia. The patient had been well until 2 months before admission, when nausea and malaise developed. His wife also reported that the patient had two episodes of transient loss of consciousness. He was sent to the emergency department of an outside hospital. At presentation, the patient had no sign of dehydration like poor skin turgor, orthostasis or decreased urine output, nor sign of volume overload including peripheral edema, ascites or elevated jugular vein pressure. Serum sodium of $102 \mathrm{mmol} / \mathrm{L}$, potassium of $3.9 \mathrm{mmol} / \mathrm{L}$ and phosphate of $1.08 \mathrm{mmol} / \mathrm{L}$ were reported. The complete blood count and the remainder of metabolic panel including serum glucose, creatinine, albumin, aminotransferase and bilirubin were within normal limits. The measured serum osmolality was 213 mosm/L. Intravenous hypertonic saline was given with an average correction rate of 8 $\mathrm{mmol} / 24 \mathrm{~h}$. Despite slow rate of correction, neurologic symptoms including restlessness and irritability developed. In the following days, the patient developed apathy, confusion and obtundation, followed by dysarthria, dysphagia, paraparesis, behavioral disturbances and disorientation. Endocrine studies revealed a serum cortisol concentration (8: 00 a.m.) of $62.4 \mathrm{nmol} /$ L (normal, 138-635 nmol/L), plasma adrenocorticotrophin (ACTH) of $1.03 \mathrm{pmol} / \mathrm{L}$ (normal, 2.2-17.6 pmol/L). The serum thyrotrophin (TSH) level was $1.37 \mathrm{mIU} / \mathrm{L}$ (normal, 0.55-4.78 $\mathrm{mIU} / \mathrm{L}$ ); serum free thyroxine (FT4) was $6.91 \mathrm{pmol} / \mathrm{L}$ (normal, 11.5-22.7 pmol/L), and serum total thyroxine (T4) was 43.0

- Received : August 21, 2014 • Revised : January 31, 2015 •Accepted : February 23, 2015

- Address for reprints : Bing Xing, M.D.

Department of Neurosurgery, Peking Union Medical College Hospital, Chinese Academy of Medical Sciences, N0.1 Shuaifuyuan, Dongcheng District, Beijing 100730, P. R. China

Tel : +86-10-69152530, Fax : +82-10-69156829, E-mail : xingbingemail@aliyun.com

- This is an Open Access article distributed under the terms of the Creative Commons Attribution Non-Commercial License (http://creativecommons.org/licenses/by-nc/3.0) which permits unrestricted non-commercial use, distribution, and reproduction in any medium, provided the original work is properly cited. 
nmol/L (normal, 65-155 nmol/L). Prednisone and levothyroxine was administered. Hyponatremia was completely corrected after that. However, neurological deficits persisted. Cranial magnetic resonance imaging (MRI) was performed and a pituitary macroadenoma (Fig. 1A, B, C) measuring 18×29×20 mm was reported. No other abnormalities including CPM or EPM were found. One week before admission, MRI was repeated and EPM was detected at bilateral basal ganglion and thalamus (Fig. 1D, E, F).

The patient was referred to our hospital because of persistent neurologic deficits. On physical exam, he was unresponsive to verbal or pain stimuli. Eye tracking and spontaneous head and limb movements were present. Pupils were of equal and normal size and reactive to light. Superficial and tendon reflexes were present and pathologic reflexes were negative. The general physical examination was normal. Metabolic panel results were normal. Hormone studies showed normal ATCH, cortisol, TSH, T4, and FT4, decreased growth hormone and insulin-like growth factor 1, normal prolactin, decreased gonadotrophin (follicle-stimulating hormone and luteinizing hormone), estrogen and testosterone. The diagnosis of nonfunctional pituitary macroadenoma, hypopituitarism and extrapontine myelinolysis were made. The patient had several episodes of restlessness and agitation with shouting and fighting lasting 10 minutes every day. Prednisone and levothyroxine supplements were continued. Quetiapine and clonazepam were given and the frequency of episodic agitation decreased gradually. Other supportive therapies including parental nutrition, aspiration precaution and physical therapy were provided.

Three weeks after admission, the patient was verbally responsive and ambulant. Dysphagia also resolved. The decision of surgery was made then based on the improved general state of the patient and the intent to remove the underlying cause of hypopituitarism and hyponatremia. Transsphenoidal surgery for pituitary adenoma was performed. Pathologically, the mass was confirmed to be nonfunctional adenoma by hematoxylin and eosin (H\&E) staining and immunohistochemical examination. Pseudorosette patterns were found on H\&E staining and nuclear division was less than one per 10 high power field. The tumor cells were scattered positive for p53 and Ki-67. And Ki-67 index was $<1 \%$. The immunostainings for ACTH, FSH, LH, PRL, TSH and GH were negative.

After discharge, the patient followed up regularly. Serum sodium was tested every week after discharge and later every month. No hyponatremia developed again after surgery. Prednisone and levothyroxine were tapered gradually. Recent hormone studies showed normal level of ACTH, cortisol, TSH, FT4 and T4. Neurological symptoms including agitation, dysar-
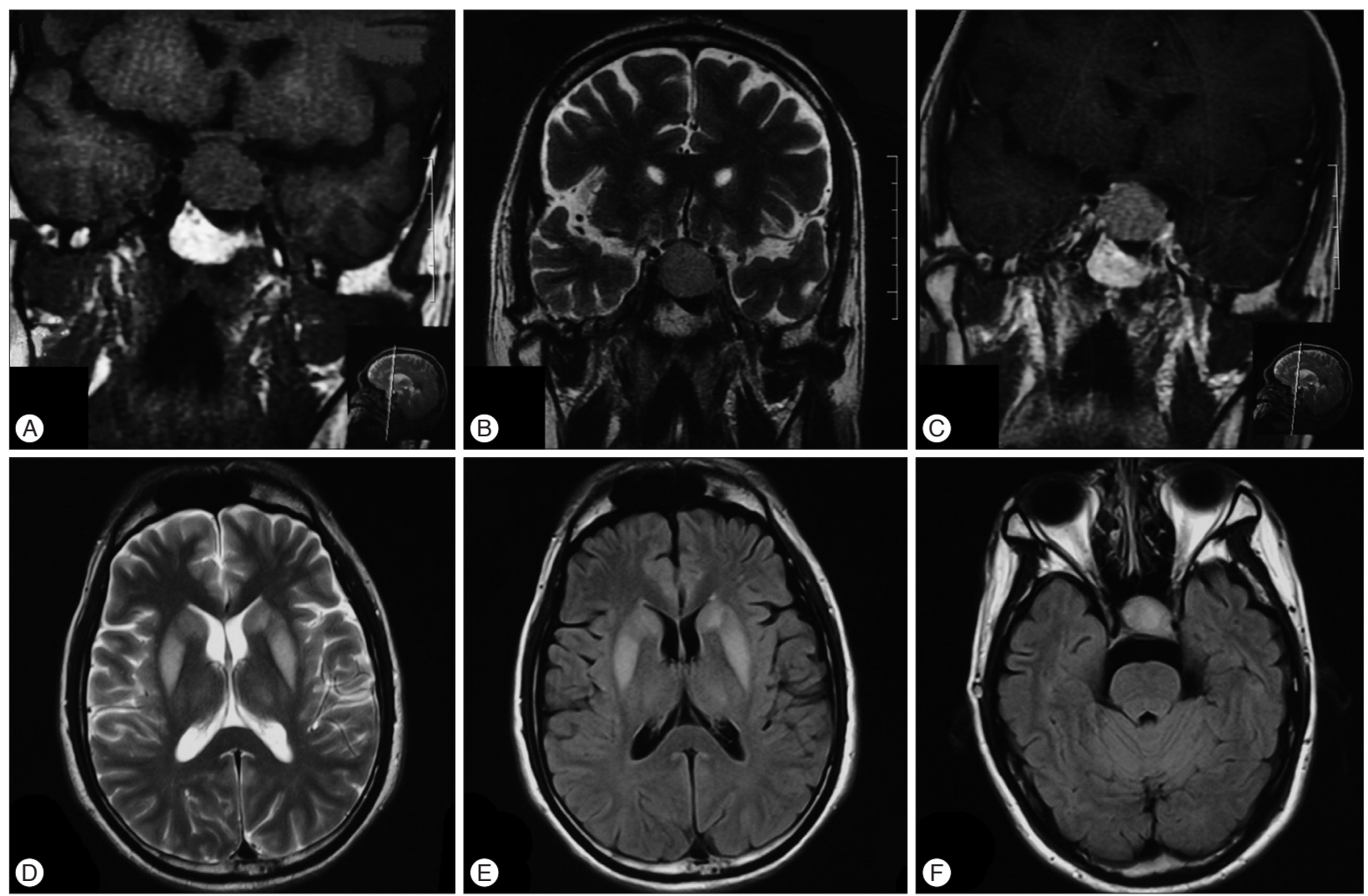

Fig. 1. Preoperative pituitary and cranial MRI. Pituitary coronal T1-weighted images (T1WI) and T2WI show an isointense lesion $(18 \mathrm{~mm} \times 29 \mathrm{~mm} \times 20$ $\mathrm{mm})$ in sella region which extends inferiorly into sphenoidal sinus and superiorly into suprasellar cistern with slight compression of optic chiasm (A and B). The mass is homogeneously enhanced (C). Cranial axial T2WI and fluid-attented inversion recovery (FLAIR) show bilateral basal ganglion and thalamus extrapontine myelinolysis ( $D$ and $E$ ). No obvious central pontine myelinolysis is found $(F)$. 

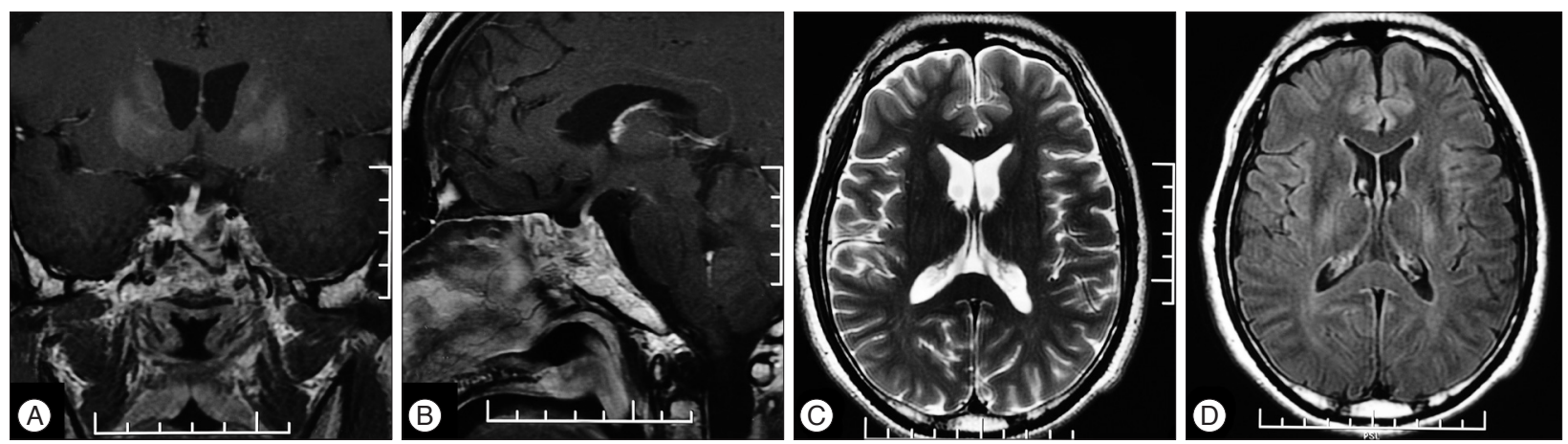

Fig. 2. Follow-up MRI 7 months after operation. Pituitary coronal and sagittal enhanced MR-images show no obvious residual adenoma (A and B). Cranial axial T2WI and FLAIR show extrapontine myelinolysis resolved at bilateral basal ganglion and thalamus (C and D).

thria, dysphagia and paraparesis resolved completely. The patient was able to resume his work as a bank accountant three months after the surgery. The latest follow-up MRI was obtained 7 months postoperatively, and these images showed demyelination at bilateral basal ganglions and thalamus resolved and pituitary adenoma resected (Fig. 2).

\section{DISCUSSION}

Among various etiologies of hyponatremia, endocrine disorders, including adrenal insufficiency and hypothyroidism, are uncommon causes which may be overlooked ${ }^{1,7,12)}$. While hyponatremia can be caused by hypopituitarism, severe hyponatremia occurring as the presenting manifestation of pituitary adenoma is rare $^{8)}$. At the onset of hyponatremia, the patient was euvolemic. He had no history of systemic disease and medication use. Given the endocrine abnormality and histology-confirmed pituitary adenoma, we propose that secondary adrenal insufficiency and hypothyroidism resulting from hypopituitarism was the underlying cause of his hyponatremia.

ODS following overly rapid correction of hyponatremia has been well recognized. Recommended rate of sodium correction is less than $10 \mathrm{mmol} / \mathrm{L}$ in any 24-hour period and less than 18 $\mathrm{mmol} / \mathrm{L}$ in any 48 -hour period to prevent ODS $^{6,17)}$. However, demyelination can occur even with this correction rate (as in our case), especially in those with other ODS risk factors ${ }^{5}$. Except for the rate of correction, serum sodium concentration at presentation and the duration of the hyponatremia are also important risk factors. On presentation, our patient had been symptomatic for 2 months and the serum sodium level was as low as $102 \mathrm{mmol} / \mathrm{L}$. Therefore, patients with more than one risk factors for ODS may still be at risk for development ODS at "normal" rates of correction and may require slower rates.

If ODS does develop despite the steps taken to avoid overly rapid correction, effective management is rare. Although reintroduction of hyponatremia, plasmapheresis and corticosteroids has been used, most evidence comes from animal models and data in humans are limited to case reports ${ }^{2,5,13)}$. The outcome of patients with ODS are often disappointing. Initial case reviews showed mortality rate as high as $50-90 \%{ }^{3)}$. A more re- cent review of 32 German patients with ODS showed a much better outcome with only $30 \%$ having irreversible and incapacitating neurological deficits ${ }^{9}$. This review suggests that patients with ODS can survive if the nonspecific secondary complications of transient illnesses such as aspiration pneumonia, deep venous thrombosis, and pulmonary embolism can be avoided.

Supportive therapy is extremely important to prevent secondary complication. Some patients with ODS can recover function after prolonged periods of severe neurologic impairment ${ }^{5,9)}$. As in our case, the patient had developed progressive neurologic symptoms for two months on admission to our hospital. These symptoms improved significantly after another three weeks of supportive therapy. The patient was able to resume the job as a banking account after surgery, indicating complete recovery from neurologic and cognitive deficits. Therefore, supportive therapy should be continued for at least six to eight weeks before concluding that the deficits are irreversible.

Surgery is the ultimate effective treatment for nonfunctioning adenoma, which not only removes the underlying cause of hypopituitarism, but also reverses or prevents vision impairment. According to a systemic review and meta-analysis evaluating the outcome of surgical treatment for nonfunctioning pituitary adenomas, pituitary hypofunction improved in as many as 30 percent after surgery ${ }^{10)}$. While for patients with EPM, appropriate surgical timing of adenoma resection is of great importance. Neurologic deficits of preoperative EPM such as decreased level of consciousness, paraparesis, dysarthria and dysphagia, posed great challenge to the transsphenoidal operation of pituitary adenoma. Risk of complications such as aspiration, respiratory failure, and deep vein thrombosis is much higher than patients without neurologic deficits ${ }^{4,9)}$. To minimize the risk of surgery and to remove the underlying cause of hyponatremia, we selectively arrange the operation after dysphagia and dysarthria resolved, and the patient became ambulant and able to obey simple commands.

\section{CONCLUSION}

We report a case of preoperative EPM with nonfunctional pituitary macroadenoma who made a great clinical recovery after 
supportive therapy and transsphenoidal adenoma resection. Outcome can be good even with prolonged periods of severe neurologic impairment. More cases are required to clarify the prognosis of ODS and identify the factors associated with better outcome.

\section{References}

1. Diederich S, Franzen NF, Bähr V, Oelkers W : Severe hyponatremia due to hypopituitarism with adrenal insufficiency : report on 28 cases. Eur J Endocrinol 148 : 609-617, 2003

2. Gankam Kengne F, Soupart A, Pochet R, Brion JP, Decaux G : Re-induction of hyponatremia after rapid overcorrection of hyponatremia reduces mortality in rats. Kidney Int 76 : 614-621, 2009

3. Gocht A, Colmant HJ : Central pontine and extrapontine myelinolysis : a report of 58 cases. Clin Neuropathol $6: 262-270,1987$

4. Kallakatta RN, Radhakrishnan A, Fayaz RK, Unnikrishnan JP, Kesavadas C, Sarma SP : Clinical and functional outcome and factors predicting prognosis in osmotic demyelination syndrome (central pontine and/or extrapontine myelinolysis) in 25 patients. J Neurol Neurosurg Psychiatry 82 : 326-331, 2011

5. King JD, Rosner MH : Osmotic demyelination syndrome. Am J Med Sci 339: 561-567, 2010

6. Laureno R, Karp BI : Myelinolysis after correction of hyponatremia. Ann Intern Med 126: 57-62, 1997

7. Liamis G, Milionis HJ, Elisaf $M$ : Endocrine disorders : causes of hyponatremia not to neglect. Ann Med 43 : 179-187, 2011

8. Lin SH, Hung YH, Lin YF : Severe hyponatremia as the presenting feature of clinically non-functional pituitary adenoma with hypopituitarism. Clin Nephrol 57 : 85-88, 2002
9. Menger H, Jörg J : Outcome of central pontine and extrapontine myelinolysis ( $\mathrm{n}=44)$. J Neurol 246 : 700-705, 1999

10. Murad MH, Fernández-Balsells MM, Barwise A, Gallegos-Orozco JF, Paul A, Lane MA, et al. : Outcomes of surgical treatment for nonfunctioning pituitary adenomas : a systematic review and meta-analysis. Clin Endocrinol (Oxf) 73 : 777-791, 2010

11. Nakano H, Ohara Y, Bandoh K, Miyaoka M : A case of central pontine myelinolysis after surgical removal of a pituitary tumor. Surg Neurol 46 : 32-36, 1996

12. Oelkers W : Hyponatremia and inappropriate secretion of vasopressin (antidiuretic hormone) in patients with hypopituitarism. N Engl J Med $321: 492-496,1989$

13. Oya S, Tsutsumi K, Ueki K, Kirino T : Reinduction of hyponatremia to treat central pontine myelinolysis. Neurology $57: 1931-1932,2001$

14. Srimanee D, Bhidayasiri R, Phanthumchinda K : Extrapontine myelinolysis in preoperative sellar region tumor : report of two cases. J Med Assoc Thai 92 : 1548-1553, 2009

15. Sterns RH, Riggs JE, Schochet SS Jr : Osmotic demyelination syndrome following correction of hyponatremia. N Engl J Med 314 : 1535-1542, 1986

16. Tosaka M, Kohga $\mathrm{H}$ : Extrapontine myelinolysis and behavioral change after transsphenoidal pituitary surgery : case report. Neurosurgery 43 : 933-936, 1998

17. Verbalis JG, Goldsmith SR, Greenberg A, Korzelius C, Schrier RW, Sterns RH, et al. : Diagnosis, evaluation, and treatment of hyponatremia : expert panel recommendations. Am J Med 126 (10 Suppl 1) : S1-S42, 2013

18. Zhao P, Zong X, Wang X, Zhang Y : Extrapontine myelinolysis of osmotic demyelination syndrome in a case of postoperative suprasellar arachnoid cyst. Case Rep Med 2012 : 679257, 2012 